\title{
Thermodynamics of Polydisperse Polymer Solutions I. Binary Mixtures
}

\author{
E. Karayianni, P. DAFNiotis, and C. PANAYiotou* \\ Chemical Process Engineering Research Institute and Department \\ of Chemical Engineering, University of Thessaloniki, \\ 54006 Thessaloniki, Greece
}

(Received July 28, 1988)

\begin{abstract}
The Lattice Fluid theory of polymer solutions, as reformulated recently, is now extended to polydisperse polymer-solvent systems. Analytical expressions are given for all basic thermodynamic quantities of the mixture as well as for the spinodals and the critical points. The theory is tested against experimental data on volumes of mixing, Flory-Huggins $\chi$ interaction parameters, spinodal curves and critical points for a number of mixtures of well characterized polydisperse polystyrene samples with cyclohexane. Comparison has also been made with experimental critical behavior of polydisperse polymer-monodisperse polymer mixtures. The agreement between theory and experiment is satisfactory. Advantages and limitations of the theory are discussed.
\end{abstract}

KEY WORDS Polydispersity / Polymer Solutions / Thermodynamics /

Lattice-Fluid Theory /

The effect of polydispersity on the thermodynamic behavior of polymer solutions have long been the subject of extensive investigations. ${ }^{1-4}$ In fact any complete thermodynamic theory of polymer systems cannot neglect the molecular weight heterogeneity of the polymer samples especially when applied to the phase behavior of these systems. In recent years the thermodynamic properties of polymer solutions are commonly interpreted with the so-called equation-of-state theories such as the new Flory theory ${ }^{5}$ and the Lattice-Fluid (LF) theory of Sanchez and Lacombe. ${ }^{6}$ Both of these theories have been proved quite successful in explaining many facets of the thermodynamic behavior of polymer solutions in terms of their "free-volume" properties or "equation-of-state" properties, and the facets of which the original well known FloryHuggins theory ${ }^{1}$ failed to explain. Although the LF theory of Sanchez and Lacombe ${ }^{6}$ is less quantitative compared to the new Flory theory ${ }^{5}$ it has one significant advantage over the latter, ${ }^{5}$ namely, it can be applied to the vapor-liquid transition.

In a recent paper $^{7}$ we reformulated the LF theory while maintaining essentially its original simplicity. The reformulated LF theory has been proved equally, if not more, successful than the new Flory theory. It became, thus, the frame for further extensions and applications to polymer-gas systems, ${ }^{8}$ random copolymer mixtures $^{9,10}$, glass transitions of polymer mixtures, ${ }^{11}$ associated solutions ${ }^{12}$ and to thermodynamics of contributions of functional groups. ${ }^{13}$

The present paper is the first in a series of paper in which the LF theory ${ }^{7}$ is extended to systems containing polydisperse polymers. It specifically deals with polydisperse polymer-

* To whom correspondence should be addressed. 
solvent and polydisperse polymer-monodisperse polymer systems and presents the complete formalism for investigations of the thermodynamic behavior of these systems. It is subsequently applied to a typical polydisperse polymer-solvent system and a typical polydisperse polymer-monodisperse polymer system.

\section{THEORY}

In this section we will present the essentials of the Lattice-Fluid formalism pertinent to the case of polydisperse polymer-solvent systems. The development will follow closely the corresponding development of monodisperse polymer-solvent systems. ${ }^{7}$ Consider, then, a mixture of $n_{1}$ molecules of a solvent, each consisting of $r_{1}$ segments, with $N_{2}$ molecules of a polydisperse polymer at temperature $T$ and external pressure $P$. The polydisperse polymer sample consists of $n_{2}$ molecules of a $r_{2}$-mer, $n_{3}$ molecules of a $r_{3}$-mer, $\cdots, n_{c}$ molecules of a $r_{\mathrm{c}}$-mer such that

$$
N_{2}=n_{2}+n_{3}+\cdots+n_{\mathrm{c}}
$$

All these molecules are arranged on a quasilattice of $N_{\mathrm{r}}$ sites, $n_{0}$ of which are empty. The LF partition function in the pressure ensemble and in its maximum term approximation may be written as

$$
Z(T, P)=\left(\frac{1}{f_{0}}\right)^{n_{0}} \prod_{m=1}^{c}\left(\frac{w_{m}}{f_{m}}\right)^{n_{m}} \exp \left[\frac{-(E+P V)}{k T}\right]
$$

where the site fractions $f_{\mathrm{i}}$ are defined by

$$
f_{0}=\frac{n_{0}}{N_{r}} ; \quad f_{i}=\frac{r_{i} n_{i}}{N_{r}}
$$

$w_{m}$ in eq 2 is the number of configurations available to an $r_{m}$-mer in the close-packed pure state.

According to the LF theory each pure $r_{\mathrm{i}}$-mer is characterized by three scaling constants $T_{i}^{*}$, $P_{i}^{*}, \rho_{i}^{*}$ for the temperature, pressure and density respectively. If $M_{i}$ is the molar mass, the hard core volume per segment, $v_{i}^{*}$, and the specific hard core volume $v_{\mathrm{sp}, i}^{*}$ are related by

$$
r_{i} v_{i}^{*}=\frac{M_{i}}{\rho_{i}^{*}}=M_{i} v_{\mathrm{sp}, i}^{*}
$$

If $s_{i}$ is the average number of external intermolecular contacts of each segment of the $r_{i}$ mer an interaction energy per segment, $\varepsilon_{i}^{*}$, can be defined as

$$
\varepsilon_{i}^{*}=\frac{s_{i}}{2} \varepsilon_{i}=P_{i}^{*} v_{i}^{*}=k \cdot T_{i}^{*}
$$

$\varepsilon_{i}$ being the interaction energy for the $i-i$ contact.

The average close-packed volume per segment in the mixture is assumed to be given by

$$
v^{*}=\sum_{i} \sum_{j} \varphi_{i} \varphi_{j} v_{i j}^{*}
$$

where $v_{i i}^{*}=v_{i}^{*}$ and

$$
v_{i j}^{*}=\xi_{i j}\left(\frac{v_{i}^{* 1 / 3}+v_{j}^{* 1 / 3}}{2}\right)^{3}
$$

$\xi_{i j}$ being a binary volume parameter (equal to one for hard spheres). The segment fraction, $\varphi_{i}$, is defined by

$$
\varphi_{i}=\frac{r_{i} n_{i}}{\sum_{j} r_{j} n_{i}}=\frac{r_{i} n_{i}}{r N}
$$

$\mathrm{N}$ being the total number of molecules in the system $\left(N=\sum_{j} n_{j}\right)$, while the surface fraction, $\vartheta_{i}$, is given by

$$
\vartheta_{i}=\frac{r_{i} n_{i} s_{i}}{\sum_{j} r_{j} n_{j} s_{j}}
$$

The total volume, $V$, of the system is given by

$$
V=N_{\mathrm{r}} v^{*}=r N \tilde{v} v^{*}
$$

where $\tilde{v}$ is the reduced volume

$$
\tilde{v}=\frac{v}{v^{*}}=\frac{1}{\tilde{\rho}}=\frac{\rho^{*}}{\rho}
$$

$v$ being the volume per segment and $\rho$ the density of the system. The potential energy, $E$, of the system is given by ${ }^{7}$ 


$$
-E=r N \tilde{\rho} \varepsilon^{*}
$$

where

$$
\varepsilon^{*}=\sum_{i} \varphi_{i} \varepsilon_{i}^{*}-\sum_{i} \sum_{j>i} \varphi_{i} \vartheta_{j} k T X_{i j}
$$

and

$$
\begin{gathered}
X_{i j}=\frac{\varepsilon_{i}^{*}+\frac{s_{i}}{s_{j}} \varepsilon_{j}^{*}-2 \sqrt{\frac{s_{i}}{s_{j}}} \varepsilon_{i j}^{*}}{k T} \\
\varepsilon_{i j}^{*}=\zeta_{i j} \sqrt{\varepsilon_{i}^{*} \varepsilon_{j}^{*}}
\end{gathered}
$$

$\zeta_{i j}$ being a binary interaction parameter (equal to one when Berthelot's rule holds).

Scaling constants for the mixture can be defined in an entirely analogous manner to that of pure components, or

$$
\varepsilon^{*}=P^{*} v^{*}=k T^{*}
$$

and the reduced quantities by

$$
\tilde{T}=\frac{T}{T^{*}}, \quad \tilde{P}=\frac{P}{P^{*}}
$$

With the above definitions the LF equation of state is given by ${ }^{6,7}$

$$
r \frac{\tilde{P} \tilde{v}}{\tilde{T}}=1-r\left[1+\frac{\ln (1-\tilde{\rho})}{\tilde{\rho}}+\frac{\tilde{\rho}}{\tilde{T}}\right]
$$

and the chemical potential of component $m$ in the mixture is given by

$$
\begin{aligned}
\frac{\mu_{m}}{k T}= & \ln \varphi_{m}+1-\frac{r_{m}}{r}+\ln \frac{\tilde{\rho}}{w_{m}}+r_{m}(\tilde{v}-1) \ln (1-\tilde{\rho}) \\
& +r_{m} \frac{\tilde{P} \tilde{v}}{\tilde{T}}\left(\frac{2 \sum_{j} \varphi_{j} v_{j m}^{*}}{v^{*}}-1\right) \\
& -\frac{r_{m}}{\tilde{v} \tilde{T}_{m}}-\frac{r_{m}}{\tilde{v}}\left[\sum_{i} \sum_{j>i} \vartheta_{i} \vartheta_{j} \frac{s_{m}}{s_{i}} X_{i j}\right. \\
& \left.-\sum_{i<m} \vartheta_{i} \frac{s_{m}}{s_{i}} X_{i m}-\sum_{j>m} \vartheta_{j} X_{m j}\right]
\end{aligned}
$$

The corresponding expression for the chemical potential of pure component $m$ is

$$
\begin{aligned}
\frac{\mu_{m}^{0}}{k T}= & \ln \frac{\tilde{\rho}_{m}}{w_{m}}+r_{m}\left(\tilde{v}_{m}-1\right) \ln \left(1-\tilde{\rho}_{m}\right) \\
& +r_{m} \frac{\tilde{P}_{m} \tilde{v}_{m}}{\tilde{T}_{m}}-\frac{r_{m}}{\tilde{v}_{m} \tilde{T}_{m}}
\end{aligned}
$$

On the basis of eq 10 the volume of mixing, $\Delta V^{M}$, is given by

$$
\Delta V^{M}=V-\sum_{i} V_{i}^{0}=r N v^{*}\left(\tilde{v}-\sum_{i} \varphi_{i} \tilde{v}_{i} \frac{v_{i}^{*}}{v^{*}}\right)
$$

Using eq 12 the heat of mixing is given by

$$
\begin{aligned}
\Delta H^{M} & =E+P V-\sum_{i}\left(E_{i}^{0}+P V_{i}^{0}\right) \\
& =r N\left[\sum_{i} \varphi_{i} \tilde{\rho}_{i} \varepsilon_{i}^{*}-\tilde{\rho} \varepsilon^{*}\right]+P \Delta V^{M}
\end{aligned}
$$

All the above equations are quite general and are applicable to any multicomponent mixture. They will now be applied to the special case of our polydisperse polymer-solvent system. A number of simplifications are possible in this case. Let us, first, define two overall fractions for the polydisperse polymer, the overall segment fraction, $\Phi_{2}$, and the overall surface fraction, $\Theta_{2}$, given by

$$
\Phi_{2}=\sum_{i>1} \varphi_{i} ; \quad \Theta_{2}=\sum_{i>1} \vartheta_{i}
$$

In order to keep manageable the complexity of the formalism we further assume that all polymer molecules, regardless of their size, are characterized by the same scaling constants $v_{2}^{*}, \varepsilon_{2}^{*}, T_{2}^{*}, P_{2}^{*}, \rho_{2}^{*}, v_{\mathrm{sp}, 2}^{*}$ and the same number of external contacts per segment, $s_{2}$. In the polymer sample we may define a number average, a weight-average and a $z$-average number of segments given respectively by

$$
\begin{aligned}
& \bar{r}_{2 N}=\frac{\sum_{j>1} n_{j} r_{j}}{N_{2}}=\frac{\bar{M}_{2 N}}{\rho_{2}^{*} v_{2}^{*}} \\
& \bar{r}_{2 w}=\frac{\sum_{j>1} n_{j} r_{j}^{2}}{\sum_{j>1} n_{j} r_{j}}=\frac{\sum_{j>1} \varphi_{j} r_{j}}{\Phi_{2}}=\frac{\bar{M}_{2 w}}{\rho_{2}^{*} v_{2}^{*}}
\end{aligned}
$$




$$
\bar{r}_{2 Z}=\frac{\sum_{j>1} n_{j} r_{j}^{3}}{\sum_{j>1} n_{j} r_{j}^{2}}=\frac{\sum_{j>1} \varphi_{j} r_{j}^{2}}{\sum_{j>1} \varphi_{j} r_{j}}=\frac{\bar{M}_{2 Z}}{\rho_{2}^{*} v_{2}^{*}}
$$

The Flory-Huggins $\chi$ interaction parameter ${ }^{1,2,5}$ can be defined in our case by

$$
\frac{\mu_{1}-\mu_{1}^{0}}{k T}=\ln \sigma_{1}+\left(1-\frac{r_{1} v_{1}^{*}}{\bar{r}_{2 N} v_{2}^{*}}\right) \sigma_{2}+\chi \sigma_{2}^{2}
$$

where the volume fractions $\sigma_{i}$ are given by

$$
\sigma_{1}=\frac{\varphi_{1} v_{1}^{*}}{\varphi_{1} v_{1}^{*}+\Phi_{2} v_{2}^{*}} ; \quad \sigma_{2}=1-\sigma_{1}
$$

Using eq 18 and 19 and the above simplifications we obtain

$$
\begin{aligned}
\chi= & \left\{\ln \frac{\varphi_{1}}{\sigma_{1}}+\Phi_{2}\left(1-\frac{r_{1}}{\bar{r}_{2 N}}\right)\right. \\
& -\sigma_{2}\left(1-\frac{r_{1} v_{1}^{*}}{\bar{r}_{2 N} v_{2}^{*}}\right)+r_{1} \tilde{\rho} X_{12} \Theta_{2}^{2}+\ln \frac{\tilde{\rho}}{\tilde{\rho}_{1}} \\
& +r_{1}(\tilde{v}-1) \ln (1-\tilde{\rho})-r_{1}\left(\tilde{v}_{1}-1\right) \ln \left(1-\tilde{\rho}_{1}\right) \\
& +r_{1} \frac{\tilde{P} \tilde{v}}{\tilde{T}}\left[\frac{2\left(\varphi_{1} v_{1}^{*}+\Phi_{2} v_{12}^{*}\right)}{v^{*}}-1\right] \\
& \left.-r_{1} \frac{\tilde{P}_{1} \tilde{v}_{1}}{\tilde{T}_{1}}-\frac{r_{1}}{\tilde{T}_{1}}\left(\tilde{\rho}-\tilde{\rho}_{1}\right)\right\} / \sigma_{2}^{2}
\end{aligned}
$$

If an entropic correction $q_{12}$ term $^{7}$ need be used, the fourth term in the previous equation should read $r_{1}\left(\tilde{\rho} X_{12}+q_{12}\right) \Theta_{2}{ }^{2}$. If we further assume that the reduced densities $\tilde{\rho}_{j}$ for the pure constituents of the polymer sample are the same (or, equivalently $r_{j} \rightarrow \infty$ for all $j>1$ ), eq 20 and 21 as well as the equation for the heat of mixing at infinite dilution, $\Delta H^{\infty}$, reduce to the corresponding expressions for the case of solvent-monodisperse polymer system. ${ }^{7}$

\section{Phase Stability}

The condition for the spinodal ${ }^{4}$ in our system may be written as

$$
\mathrm{D}=\left|\begin{array}{cccc}
g_{22} & g_{23} & \cdots & g_{2 \mathrm{c}} \\
g_{32} & g_{33} & \cdots & g_{3 \mathrm{c}} \\
\cdot & \cdot & \cdots & \cdot \\
g_{\mathrm{c} 2} & g_{\mathrm{c} 3} & \cdots & g_{\mathrm{cc}}
\end{array}\right|=0
$$

where

$$
g_{j i}=\left[\frac{\partial}{\partial \varphi_{j}}\left(\frac{\partial g}{\partial \varphi_{i}}\right)_{T, P, \varphi_{k \neq 1, i}}\right]_{T, P, \varphi_{m \neq 1, j}}
$$

and

$$
g=\frac{G}{r N k T}
$$

$G$ being the Gibbs free energy of the system $(G=-k T \ln Z)$. By using the simplifying assumptions reported previously eq 29 reduces to

$$
\begin{aligned}
\frac{1}{\varphi_{1} r_{1}} & +\frac{1}{\Phi_{2} \bar{r}_{2 w}}-2 \frac{\vartheta_{1}}{\varphi_{1}} \frac{\Theta_{2}^{2}}{\Phi_{2}^{2}}\left(\tilde{\rho} X_{12}+q_{12}\right) \\
& +2 \frac{\tilde{P} \tilde{v}}{k T}\left(v_{1}^{*}+v_{2}^{*}-2 v_{12}^{*}\right)-\tilde{\rho} Q_{2} \psi_{2}=0
\end{aligned}
$$

where

$$
\begin{aligned}
& Q_{2}=\beta P^{*} \tilde{T} \psi_{2} \\
& \psi_{2}=\tilde{\rho} \lambda_{2}+\frac{P \tilde{v}}{k T} D_{2}+\frac{1}{r_{1}} \\
& \lambda_{2}=\frac{1}{\tilde{T}_{2}}-\frac{1}{\tilde{T}_{1}}+\left(\Theta_{2}^{2}-\vartheta_{1}^{2} \frac{s_{2}}{s_{1}}\right) X_{12} \\
& D_{2}=2\left[\varphi_{1}\left(v_{12}^{*}-v_{1}^{*}\right)+\Phi_{2}\left(v_{2}^{*}-v_{12}^{*}\right)\right]
\end{aligned}
$$

and

$$
\beta P^{*}=\frac{\tilde{v}^{2}}{\tilde{T} \tilde{v}\left(\frac{1}{\tilde{v}-1}+\frac{1}{r}\right)-2}
$$

At the critical point the following condition should be satisfied in addition to eq 29 


$$
Y=\left|\begin{array}{cccc}
\frac{\partial \mathrm{D}}{\partial \varphi_{2}} & \frac{\partial \mathrm{D}}{\partial \varphi_{3}} & \cdots & \frac{\partial \mathrm{D}}{\partial \varphi_{\mathrm{c}}} \\
g_{32} & g_{33} & \cdots & g_{3 \mathrm{c}} \\
\cdot & \cdot & \cdots & \cdot \\
g_{\mathrm{c} 2} & g_{\mathrm{c} 3} & \cdots & g_{\mathrm{cc}}
\end{array}\right|=0
$$

With the previous simplifying assumptions eq 38 reduces to

$$
\begin{aligned}
\frac{1}{\varphi_{1}^{2} r_{1}} & -\frac{\bar{r}_{2 Z}}{\Phi_{2}^{2} \bar{r}_{2 w}^{2}}+6 \frac{\vartheta_{1}}{\varphi_{1}} \frac{\Theta_{2}^{2}}{\Phi_{2}^{2}}\left\{\left(\frac{\Theta_{2}}{\Phi_{2}}-\frac{\vartheta_{1}}{\varphi_{1}}\right)\right. \\
& \left.\times\left(\tilde{\rho} X_{12}+q_{12}\right)-\tilde{\rho}^{2} Q_{2} X_{12}\right\} \\
& +\tilde{\rho}^{2} Q_{2}^{2}\left\{\left(\frac{1}{\bar{r}_{2 N}}-\frac{1}{r_{1}}\right)-4 \tilde{\rho} \lambda_{2}\right. \\
& \left.-Q_{2}\left(\frac{2 \tilde{\rho}^{2}}{\tilde{T}}-\frac{1}{(\tilde{v}-1)^{2}}\right)\right\} \\
& -2 \frac{P Q_{2}}{k T}\left\{3\left(v_{1}^{*}+v_{2}^{*}-2 v_{12}^{*}\right)-\tilde{\rho} Q_{2} D_{2}\right\}=0
\end{aligned}
$$

\section{APPLICATIONS}

In this section we will apply the above formalism, first, to a typical example of a polydisperse polymer-solvent system, namely, the polystyrene-cyclohexane mixture. This particular system became the subject of numerous intensive investigations over many years ${ }^{3.14-21}$ resulting in abundantly confirmed extensive experimental data. Scholte ${ }^{20}$ has reported spinodal data for polystyrene in cyclohexane from light scattering measurements and for three well characterized polydisperse polymer samples. Koningsveld et al. ${ }^{19}$ on the other hand have reported experimental data on critical temperatures and critical compositions for a number of mixtures of cyclohexane with polystyrene samples of a wide variety of molecular weight distributions all produced by mixing proprietary sharp fractions of polystyrene. In what follows the present theory will be applied to these two sets of data.
In order to apply the theory to the system of Cyclohexane (1)-Polystyrene (2) we need first the LF scaling constants for the pure components. The LF constants have been obtained from the volumetric properties of pure compounds at $25^{\circ} \mathrm{C}$ reported by Höcker et al. ${ }^{18,22}$ and are: $T_{1}^{*}=502 \mathrm{~K}, P_{1}^{*}=417 \mathrm{MPa}, \rho_{1}^{*}=886$ $\mathrm{kg} \mathrm{m}^{-3} ; T_{2}^{*}=632 \mathrm{~K}, P_{2}^{*}=466 \mathrm{MPa}$ and $\rho_{2}^{*}=$ $1139 \mathrm{~kg} \mathrm{~m}^{-3}$. Following Saeki et al. ${ }^{21}$ the value 1.68 was assigned to the ratio $s_{1} / s_{2}$. The two adjustable binary parameters $\zeta_{12}$ and $q_{12}$ of the theory have been determined from a least-square fit of the above two sets of data. ${ }^{19,20}$ The values of the binary parameters so obtained are: $\zeta_{12}=1.0349$ and $q_{12}=-0.058$ both dimensionless. In Table I are shown the experimental $^{19}$ and the calculated critical temperatures and critical compositions with both the present theory and the five-parameter theory of Kennedy et al. ${ }^{3}$ As observed the performance of the present theory is gratifying. In Figure 1 are shown experimental ${ }^{20}$ and calculated spinodals for three systems of different molecular weight and molecular weight distribution polystyrenes. Again the performance of the theory is quite satisfactory. In the same Figure are shown experimental ${ }^{27}$ and calculated spinodals (dashed curves a and b) for two heterodisperse Polystyrene-Cyclohexane systems. Molecular weight averages of the two Polystyrene samples are as following: curve a: $\bar{M}_{n}=54000, \bar{M}_{w}=110000$, $\bar{M}_{z}=173000 ;$ curve b: $\bar{M}_{n}=45000, \quad \bar{M}_{w}=$ 165000, $\bar{M}_{z}=432000$. The experimental data are reproduced rather satisfactorily with the above set of binary parameters.

Using the above binary parameters $\left(\zeta_{12}\right.$ and $q_{12}$ ) we have calculated the predictions of the theory for the $\chi$ interaction parameter at $25^{\circ} \mathrm{C}$. In Figure 2 are shown the experimental and predicted $\chi$ interaction parameters. As observed experiment and theory are in very good agreement. Volumes of mixing over extended ranges of composition can be reproduced easily by the LF model $^{7}$ by slightly adjusting the volume parameter $\xi_{12}$. In the present case the 
Table I. Critical temperatures and critical compositions for the system cyclohexane(1)-polystyrene(2)

\begin{tabular}{|c|c|c|c|c|c|c|c|c|}
\hline \multicolumn{5}{|c|}{ Experimental } & \multicolumn{4}{|c|}{ Calculated } \\
\hline \multirow{2}{*}{$\bar{M}_{n} \times 10^{-3}$} & \multirow{2}{*}{$\bar{M}_{w} \times 10^{-3}$} & \multirow{2}{*}{$\bar{M}_{z} \times 10^{-3}$} & \multirow{2}{*}{$T_{\mathrm{c}} / \mathrm{K}$} & \multirow{2}{*}{$M_{\mathrm{c}_{2}}$} & \multicolumn{2}{|c|}{ Kennedy et al. ${ }^{3}$} & \multicolumn{2}{|c|}{ This work } \\
\hline & & & & & $T_{\mathrm{c}} / \mathrm{K}$ & $W_{\mathrm{c}_{2}}$ & $T_{\mathrm{c}} / \mathrm{K}$ & $W_{\mathrm{c}_{2}}$ \\
\hline 27 & 35.4 & 45.5 & 284.6 & 0.179 & - & - & 287.9 & 0.196 \\
\hline 49 & 51 & 55 & 288.9 & 0.146 & 286.5 & 0.216 & 290.3 & 0.153 \\
\hline 55 & 61.5 & 70.5 & 290.5 & 0.143 & 289.0 & 0.159 & 291.4 & 0.143 \\
\hline 91 & 93 & 96 & 293.7 & 0.117 & 290.3 & 0.169 & 293.8 & 0.110 \\
\hline 154 & 166 & 181 & 296.6 & 0.099 & 293.1 & 0.125 & 296.5 & 0.083 \\
\hline 200 & 286 & 438 & 298.7 & 0.095 & 296.4 & 0.119 & 298.5 & 0.073 \\
\hline 210 & 346 & 550 & 300.2 & 0.092 & 297.8 & 0.174 & 299.2 & 0.068 \\
\hline 375 & 394 & 423 & 300.7 & 0.070 & 299.6 & 0.090 & 299.8 & 0.052 \\
\hline 490 & 527 & 593 & 301.2 & 0.064 & 300.3 & 0.089 & 300.7 & 0.046 \\
\hline 1250 & 1500 & 1700 & 303.2 & 0.042 & 302.0 & 0.065 & 303.2 & 0.027 \\
\hline
\end{tabular}

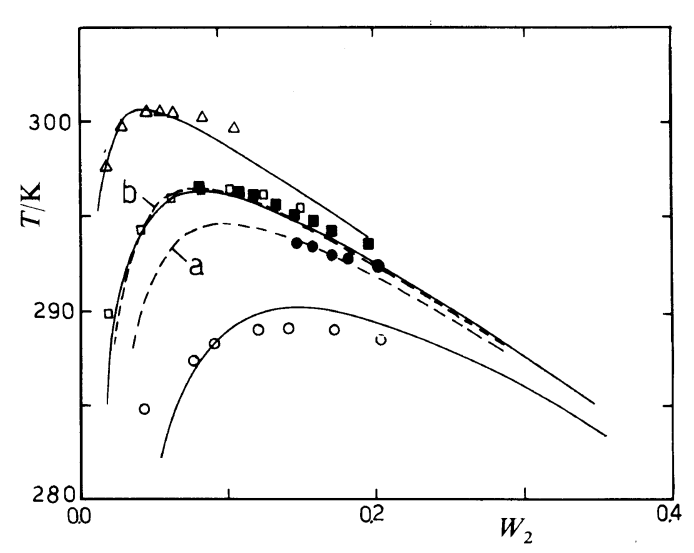

Figure 1. Experimental ${ }^{20}$ and calculated spinodals (solid curves) for the system cyclohexane(1)-polystyrene(2) vs. the weight fraction of polystyrene. Circles are for $\bar{M}_{n}=49000 ;$ squares for $\bar{M}_{n}=154000$ and triangles for $\bar{M}_{n}=435000$. Filled circles and rectangles are experimental data of Derham et al. ${ }^{27}$ Corresponding dashed curves $a$ and $b$ are calculated as explained in the text.

volumes of mixing reported by Höcker et al. ${ }^{18}$ are reproduced to within experimental error by setting $\xi_{12}=1.0049$.

The critical points reported by Koningsveld et al. ${ }^{19}$ and used in this work are of the UCST type (upper critical solution temperature). In addition to UCST behavior the cyclohexanepolystyrene system exhibits also the common LCST behavior (lower critical solution temperature). Critical temperatures of this latter

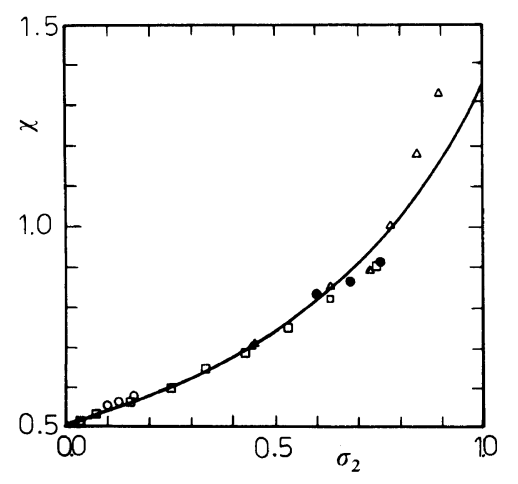

Figure 2. The reduced residual chemical potential $\chi$ for the system cyclohexane(1)-polystyrene(2). The solid curve is predicted by the present theory. Open circles are from osmotic pressure measurements of Palmen ${ }^{23}$ at $26^{\circ} \mathrm{C}$. Filled circles represent vapor pressure measurements of Schmoll and Jenckel ${ }^{24}$ at $25^{\circ} \mathrm{C}$. Triangles show vapor pressure results of Krigbaum and Geymer ${ }^{25}$ at $24^{\circ} \mathrm{C}$ and squares indicate values of Scholte ${ }^{26}$ obtained by equilibrium ultracentrifugation at $30^{\circ} \mathrm{C} . \sigma_{2}$ is the hard core volume fraction of polystyrene. ${ }^{7}$

type ranging from $180^{\circ} \mathrm{C}$ to $220^{\circ} \mathrm{C}$ have been reported in the literature. ${ }^{14-17,28}$ The present model does predict a LCST for our system. It is not, however, expected that the pure component parameters and the binary parameters which have been determined at about $300 \mathrm{~K}$ to be appropriate at $500 \mathrm{~K}$. On the basis of the volumetric properties reported by Höcker et $a l .{ }^{18}$ the following pure component LF pa- 
Table II. LCST's for the system cyclohexane(1)polystyrene(2)

\begin{tabular}{cccc}
\hline \multicolumn{2}{c}{ Experimental 17, 28} & & Calculated \\
\cline { 1 - 2 } $\bar{M}_{w} \times 10^{-4}$ & $T_{\text {cr }} / \mathrm{K}$ & & $T_{\mathrm{cr}} / \mathrm{K}$ \\
\hline 3.70 & 510.9 & & 508.3 \\
9.72 & 502.1 & & 500.5 \\
20.0 & 496.9 & & 496.6 \\
40.0 & 494.7 & & 493.9 \\
67.0 & 491.7 & & 492.4 \\
270 & 488.6 & & 489.9 \\
333 & 488.2 & & 489.7 \\
\hline
\end{tabular}

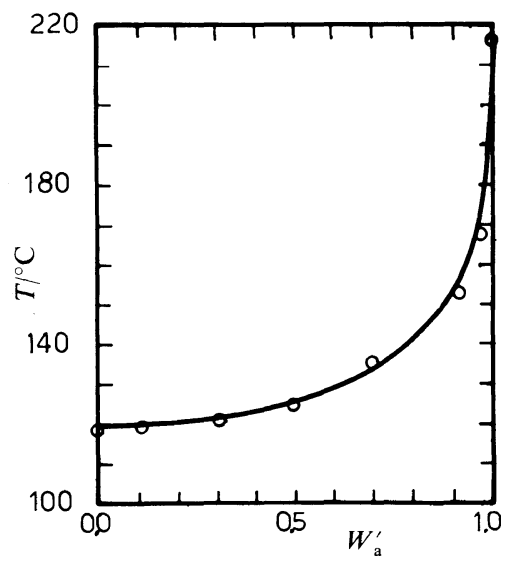

Figure 3. Influence of polydispersity on the phase behavior in the system PVME(1)-polydisperse polystyrene(2). Circles are experimental cloud points ${ }^{29}$ of a mixture $50 \mathrm{wt} \%$ in polystyrene. Solid curve is the corresponding calculated spinodal. $W_{\mathrm{a}}^{\prime}$ is the weight fraction of constituent $a$ in the polydisperse polystyrene sample.

rameters have been obtained at $200^{\circ} \mathrm{C}$ : $T_{1}^{*}=592 \mathrm{~K}, P_{1}^{*}=384 \mathrm{MPa}, \rho_{1}^{*}=815 \mathrm{~kg} \mathrm{~m}^{-3}$; $T_{2}^{*}=806 \mathrm{~K}, P_{2}^{*}=412 \mathrm{MPa}, \rho_{2}^{*}=1081 \mathrm{~kg} \mathrm{~m}^{-3}$. With these pure component parameters and the same set of binary parameters we calculated the LCST's for our system. Comparison with experiment ${ }^{17,28}$ is made in Table II. As observed the performance of the model is again quite satisfactory.

The formalism presented in this work is equally valid to the case of monodisperse polymer(1)-polydisperse polymer(2) systems. It is worth comparing, then, our model with experimental data pertinent to this type of systems. Nishi and $\mathrm{Kwei}^{29}$ have reported experimental cloudpoints for mixtures of monodisperse Poly(vinyl methyl ether) (PVME) (1)-polydisperse Polystyrene (2) 50\% wt. in Polystyrene. Polystyrene samples were obtained by mixing two monodisperse samples a $(M=10000)$ and $b(M=110000)$. In Figure 3 are compared the experimental data with the calculated spinodals for mixtures $50 \%$ wt in Polystyrene. Pure component parameters and binary parameters for this system have been obtained from our previous work. ${ }^{7}$ The trend of LCST with the polydispersity of the Polystyrene sample is well reproduced by the present model.

\section{DISCUSSION AND CONCLUSIONS}

Inspite the satisfactory performances reported in the previous section the LF model has inherent limitations as any other similar freevolume model for polymer solutions. The oversimplistic approach of the present work ( $\tilde{\rho}_{j}$ the same for all pure constituents of the polydisperse polymer or $r_{j} \rightarrow \infty$ for all $j>1$ ) is not expected to hold for highly polydisperse polymer-solvent systems. Derham et al. ${ }^{27}$ and Scholte $^{30}$ have conducted a series of carefull experimental pulse induced critical scattering measurements for highly polydisperse Polystyrene-Cyclohexane systems. Scholte's Polystyrene samples were characterized by the same $\bar{M}_{w}(100000)$ but with an $\bar{M}_{n}$ ranging from 21600 to 70000 . In a temperature $v s$. composition diagram the experimental spinodal curves are departing from each other. In the studied composition range the spinodals for the two extreme $\bar{M}_{n}$ values have shown a maximum deviation of $c a .1 .7$ degrees centigrade. The present model, when using the pure component and binary parameters of the previous section, calculates a maximum deviation of only 0.3 degrees centigrade in the same composition range. Agreement between theory and experiment can be reached by slightly 
changing the binary parameter $\zeta_{12}$ as following: For the samples with $\bar{M}_{n}$ equal to 70000 , 38000 and $21600, \zeta_{12}$ must be equal to 1.03487 , 1.03500 and 1.03505 respectively. These changes in $\zeta_{12}$ correspond to small changes in $\mathrm{X}_{12}$ which can easily by obtained by changes in $T_{2}^{*}$ of pure polymer by a few degrees centigrade or equivalently by a small change in the thermal expansion coefficient of the polymer. ${ }^{31}$ Unfortunately the volumetric properties of the different polydisperse Polystyrene samples, from which one could get their characteristic LF constants, are not available.

The change of $\zeta_{12}\left(\right.$ or $\mathrm{X}_{12}$ ) with $\bar{M}_{n}$, although successful, is essentially a rather empirical approach. Other more fundamental approaches such as the group-contribution approach $^{13}$ may be applied to polydisperse systems and may lead to a formalism with manageable complexity. Such an approach, however, can be useful only after the establishment of a rich database with the characteristic group and intergroup parameters.

In any case, and because of its simplicity, the present model may form the basis for more refined treatments of the phase behavior (UCST, LCST, liquid-vapor transition) of polymer solutions.

\section{REFERENCES}

1. P. J. Flory, "Principles of Polymer Chemistry," Cornell Univ. Press, Ithaca, N. Y., 1953.

2. H. Tompa, "Polymer Solutions," Butterworths, London, 1956.

3. J. W. Kennedy, M. Gordon, and R. Koningsveld, $J$. Polym. Sci., C, 39, 43 (1972).

4. M. Kurata, "Thermodynamics of Polymer Solutions," Harwood Academic Publishers, New York, N. Y., 1982.
5. P. J. Flory, R. A. Orwoll, and A. Vrij, J. Am. Chem Soc., 86, 3507, 3515 (1964).

6. I. Sanchez and R. Lacombe, Macromolecules, 11, 1145 (1978).

7. C. Panayiotou, Macromolecules, 20, 861 (1987).

8. C. Panayiotou, Makromol. Chem., 187, 2867 (1986).

9. C. Panayiotou, Makromol. Chem., 188, 2733 (1987).

10. P. Dafniotis, E. Karayianni, and C. Panayiotou, Makromol. Chem., in press.

11. C. Panayiotou, Polym. J., 18, 895 (1986).

12. C. Panayiotou, J. Phys. Chem., 92, 2960 (1988).

13. C. Panayiotou, Polym. J., 20, 603 (1988).

14. G. Allen and C. H. Baker, Polymer, 6, 181 (1965).

15. C. H. Baker, C. S. Clemson, and G. Allen, Polymer, 7, 525 (1966).

16. A. A. Tager, A. A. Anikeyeva, V. M. Andreyeva, T. Ya. Gumarova, and L. A. Chernoskutova, Polym. Sci. U.S.S.R., 10, 1926 (1968).

17. A. A. Tager, V. M. Andreyeva, S. A. Vshivkov, and I. S. Tjukova, J. Polym. Sci., Polym. Symp., 61, 283 (1977).

18. H. Höcker, H. Shih, and P. J. Flory, Trans. Faraday Soc., 67, 2275 (1971).

19. R. Koningsveld, L. A. Kleintjens, and A. R. Schultz, J. Polym. Sci., A-2, 8, 1261 (1970).

20. Th. G. Scholte, J. Polym. Sci., A-2, 9, 1553 (1971).

21. S. Saeki, J. C. Holste, and D. C. Bonner, J. Polym. Sci., Phys. Ed., 19, 307 (1981).

22. H. Höcker, G. J. Blake, and P. J. Flory, Trans. Faraday Soc., 67, 2251 (1971).

23. K. J. Palmen, Thesis, Technische Hochschule, Aachen, W. Germany, 1965.

24. K. Schmoll and E. Jenckel, Z. Elektrochem., 60, 756 (1956).

25. W. R. Krigbaum and D. O. Geymer, J. Am. Chem. Soc., 81, 1859 (1959).

26. Th. G. Scholte, J. Polym. Sci., A-2, 8, 841 (1970).

27. K. Derham, J. Goldsbrough, and M. Gordon, Pure Appl. Chem., 38, 97 (1974).

28. S. Saeki, N. Kuwahara, S. Konno, and M. Kaneko, Macromolecules, 6, 246 (1973).

29. T. Nishi and T. K. Kwei, Polymer, 16, 285 (1975).

30. Th. G. Scholte, J. Polym. Sci., C, 39, 281 (1972).

31. C. Panayiotou, in "Integration in Polymer Science and Technology," L. Kleintjens and P. Lemstra, Ed., Elsevier Applied Science Publishers, London, 1988. 